\title{
Near-Infrared Fluorescence Lymph Node Navigation Using Indocyanine Green for Gastric Cancer Surgery
}

\author{
Seong-Ho Kong, M.D., Ph.D. ', Seong-Woo Bae, M.S. ${ }^{2}$, Yun-Suhk Suh, M.D., Ph.D. ', Hyuk-Joon Lee, M.D., Ph.D. ${ }^{1,2}$, \\ Han-Kwang Yang, M.D., Ph.D. ${ }^{1,2}$ \\ ${ }^{1}$ Department of Surgery, Seoul National University Hospital, ${ }^{2}$ Cancer Research Institute, Seoul National University, Seoul, Korea
}

\begin{abstract}
Near-infrared (NIR) fluorescence imaging is a promising method for image-guided surgery, providing robust functional images with relatively good cost-effectiveness. A cyanine vital dye indocyanine green (ICG) is a safe NIR fluorophore emitting $800 \sim 840 \mathrm{~nm}$ of light and has been used in numerous surgical procedures. The technique has been applied to lymph node navigation of gastric cancer surgery with an expectation of better visualization of lymphatic structures without any risk of radio-hazard compared with a "dual method" using both vital dyes and radioisotopes. Given the characteristics of ICG, such as fast distribution and quenching effect, diluted concentrations, such as $0.05 \sim 0.1 \mathrm{mg} / \mathrm{ml}$, are thought to be optimal for sentinel node navigation. Injection into the subserosal layer is feasible; however, endoscopic submucosal injection has advantages of improved accuracy of the injection site and feasibility of injection one day prior to surgery; these advantages are preferred by some investigators due to a smaller number of sentinel nodes compared with injection in the operation theatre. The technology requires evaluation of the sensitivity and specificity, as well as the non-inferiority, compared with the dual method in a large cohort for justification as a safe node navigation method.
\end{abstract}

Received March 8, 2018

Revised April 18, 2018

Accepted May 9, 2018

Corresponding author

Seong-Ho Kong

Department of Surgery, Seoul

National University Hospital, 101

Daehak-ro, Jongno-gu, Seoul 03080,

Korea

Tel: $+82-2-2072-4199$

Fax: +82-2-766-3975

E-mail: seongho.kong@snu.ac.kr ORCID:

http://orcid.org/0000-0002-3929-796X

Keywords: Near-infrared, Indocyanine green, Sentinel lymph node biopsy, Stomach neoplasms

This is an Open Access article distributed under the terms of the Creative Commons Attribution Non-Commercial License (http:// creativecommons.org/licenses/by-nc/4.0/) which permits unrestricted non-commercial use, distribution, and reproduction in any medium, provided the original work is properly cited.

Copyright $@ 2018$ The Journal of Minimally Invasive Surgery. All rights reserved.

\section{INTRODUCTION}

According to the increasing needs and interest in precision surgery, which is characterized by individual tailored surgical strategies, image-guided surgery is receiving increased attention as the most intuitive and strong methodology to implement precision surgery. ${ }^{1}$ Computer-based augmented reality, which overlays the virtual images reconstructed from preoperative CT scan or MRI on the operative visual field, is a technology used to optically navigate the surgical view, and its applications are expanding, especially to surgeries of rigid organs, such as orthopedic surgery, neurosurgery, and dental surgery. ${ }^{2,3}$ Its practical usage in abdominal surgery has not been as rapidly implemented compared with rigid organ surgery given the increased complexity of non-rigid registration between the virtual images and optical images due to numerous factors, resulting in deformities of the organ during the surgery. In addition, the need for additional time, human power, and resources to construct virtual images and overlaying procedures has been limiting.

In contrast, near-infrared (NIR) imaging using indocyanine green (ICG) has been independently developed for functional imaging of location and patency of vascular structures in neuro-, ophthalmologic, and vascular surgeries since $1980 \mathrm{~s}^{4-6}$ The wavelength of NIR light is approximately 700 1,000 nm. This range is the least absorbed by blood or water; thus, this "optical window" has been regarded as the best wavelength of imaging to provide the deepest penetration of the signal. ${ }^{78}$ Indocyanine 
green is an FDA-approved vital dye for liver function tests and exhibits good characteristics as a fluorophore for NIR images. NIR imaging has been applied to various procedures in general surgery, providing functional information of the perfusion of the organs, visualization of the biliary tract and hepatic tumors, and margins of the anatomic segments of the liver. $^{9-13}$

ICG has been used as a vital dye to visualize sentinel lymph nodes, which are observed by the naked eye. ${ }^{14}$ ICG can provide better sensitivity and depth penetration when used together with NIR imaging systems, which are composed of excitation light sources and special filters and cameras optimized for the NIR wavelength of light. ${ }^{15,16}$ Compared with radioisotopes, which are additional tools used for sentinel node navigation, ICG has advantages that can avoid special safety protocols necessary for the use of radioactive dyes. Therefore, NIR imaging using ICG is under active investigation as an effective and safe tool for lymph node navigation in oncologic surgeries, including cancers of the stomach, esophagus, colon, and gynecologic organs. ${ }^{17-19}$

This article is intended to review previous studies of NIR fluorescent imaging using ICG in lymph node navigation in gastric cancer surgery with a special focus on when and how to inject the ICG.

\section{ICG AND NIR FLUORESCENT OPTICAL SYSTEMS}

ICG was approved by the Food and Drug Administration (FDA) for liver function tests in 1958 given its almost exclusive metabolization by the liver. It is one of the safest vital dyes with only very rare reports of hypersensitivity reactions. ${ }^{7}$ ICG belongs to cyanine dyes, which structurally contain two rings and double bonds between them. The length of the double bonds determines the wavelength of the emitted signal. ICG is excited at 700 800 $\mathrm{nm}$, and the emitted maximal signal is $800 \sim 840 \mathrm{~nm}^{20}$

Previously, tracers with high photon yields, such as quantum dots, were suggested as tracer fluorophores for NIR imaging. Strong excitation light and the usage of quantum dots can result in very deep penetration signal depths; however, possible harmful effects of excessive excitation light and heavy metals in quantum dots have limited their application in human surgeries. In contrast, ICG exhibits a great safety profile. However, the quantum yield is relatively low, thus the penetration depth for practical usage seems to be a maximum of $0.5 \mathrm{~cm}$.

Of note, ICG has not been approved for NIR imaging by the FDA until recently, and most of the studies used ICG for offlabeled indications.

Initial studies used NIR imaging systems designed for open surgeries. Many gastric surgery researchers used a prototype system based on the photodynamic eye (PDE) system (Hamamatsu Photonics, Hamamatsu, Japan). ${ }^{21-26}$ This system only shows NIR imaging.

Yoshida et al. used the Hyper Eye Medical System (HEMS; Mizuho Ikakogyo Co., Ltd, Tokyo, Japan), which simultaneously provides both NIR images and white light images, ${ }^{27}$ and Frangioni and Tummer et al. developed systems for open surgeries, including the Mini-Fluorescence-Assisted Resection and Exploration (Mini-FLARE ${ }^{\mathrm{TM}}$ ) image-guided surgery system. $^{28}$

The Novadaq company commercialized the laparoscopic NIR imaging system Pinpoint ${ }^{\mathrm{TM}}$ and its DaVinci version Fire$\mathrm{fly}^{\mathrm{TM}}$ system. ${ }^{29}$ Both systems provide fused images from white light and NIR imaging. ${ }^{30}$

Other companies have reported prototypes of laparoscopic systems from other companies, ${ }^{31}$ and several laparoscopic systems, including the D-light ${ }^{\mathrm{TM}}$ system (Karl-Storz, Tuttlingen, Germany), AIM ENV ${ }^{\mathrm{TM}}$ system (Stryker, San Jose, CA, USA), and $\mathrm{IR}^{\mathrm{TM}}$ system (Olympus, Tokyo, Japan), are currently available in the market (Fig. 1).

\section{INJECTION OF ICG INTO THE STOMACH}

Most of the locations of the injection for sentinel lymph node navigation involve the four quadrants adjacent to the gastric cancer: proximal, distal, and two lateral areas. For small tumors, which are usually indicated for sentinel lymph node navigation, 4 injection sites are regarded as sufficient to cover the entire lymphatic flow from primary tumors because ICG spreads greater than $1 \mathrm{~cm}$ when injected into the gastric wall. However, additive injection may be required for larger tumors when the ICG injection cannot cover entire margin of the tumor (Fig. 2). ${ }^{25}$

ICG can be injected either into the subserosal layer by the surgeon or into the submucosal layer via endoscopic needle. Ryu et al. compared submucosal injection and subserosal injection using the vital dye isosulfan blue and reported no differences in the detection rate, number of sentinel lymph nodes, and sensitivity. ${ }^{32}$ No study has compared two access routes in the NIR system, and both methods have been used.

Subserosal injection seems to be beneficial given that the surgeon can control the injection by her/himself. .1,23-27,30,31 $^{\mathrm{Ad}-}$ ditionally, in my personal opinion, ICG injected into the subserosal layer reaches to the lymphatic pathway more quickly compared with ICG injected into the submucosal layer. However, the location of the primary tumor is frequently difficult to identify using the external view unless the tumor is too advanced to be easily visualized. Some areas may be technically demanding to inject, especially in laparoscopic surgery. 

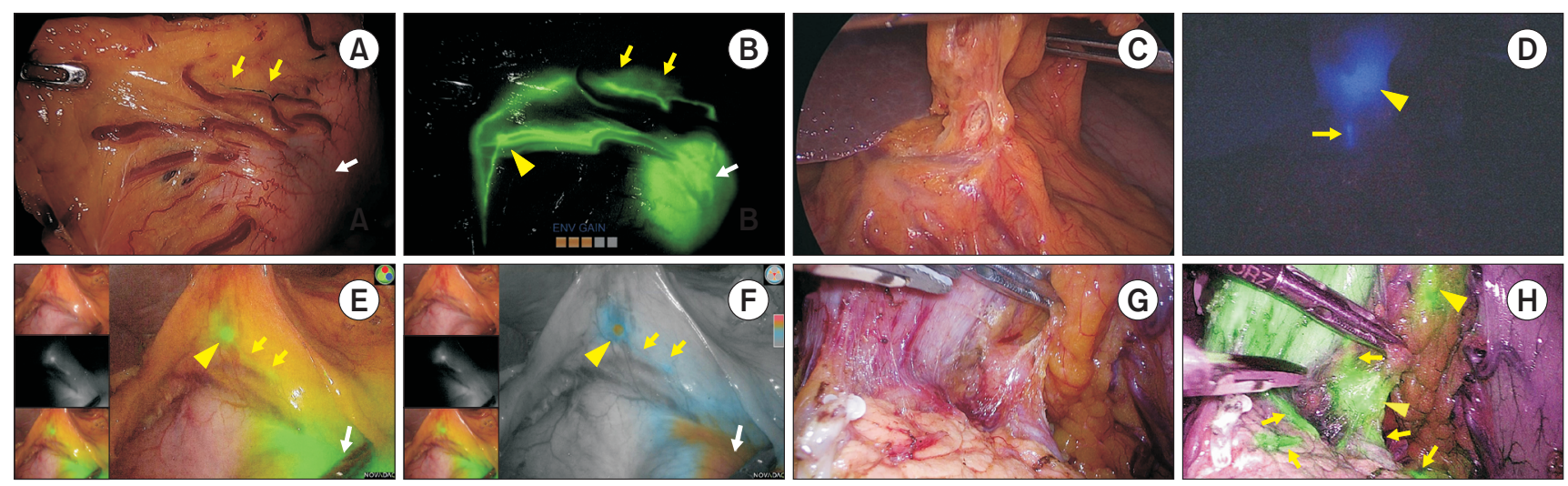

Fig. 1. Examples of lymph node navigation using various imaging systems. Yellow arrowhead: Iymph node. Yellow arrow: lymphatic channel. White arrow: injection site. After an injection of $2.5 \mathrm{mg} / \mathrm{ml}$ of indocyanine green, only one green lymphatic channel is observed by white light view (A), however, multiple lymphatic channels as well as a lymph node are visualized by AIM system ENV ${ }^{T M}$ mode (Stryker, San Jose, CA, USA) (B). After injection of 0.1 $\mathrm{mg} / \mathrm{ml}$ of indocyanine, lymphatic channels and lymph nodes are visualized in the D-light ${ }^{\mathrm{TM}}$ system (Karl-Storz, Tuttlingen, Germany) IC, D), Pinpoint fluorescence mode (E) and color segmented fluorescence mode (F) in Pinpoint ${ }^{\mathrm{TM}}$ system (Novadaq, Canada), and IIT ${ }^{\mathrm{TM}}$ system (Olympus, Tokyo, Japan) (G, H).
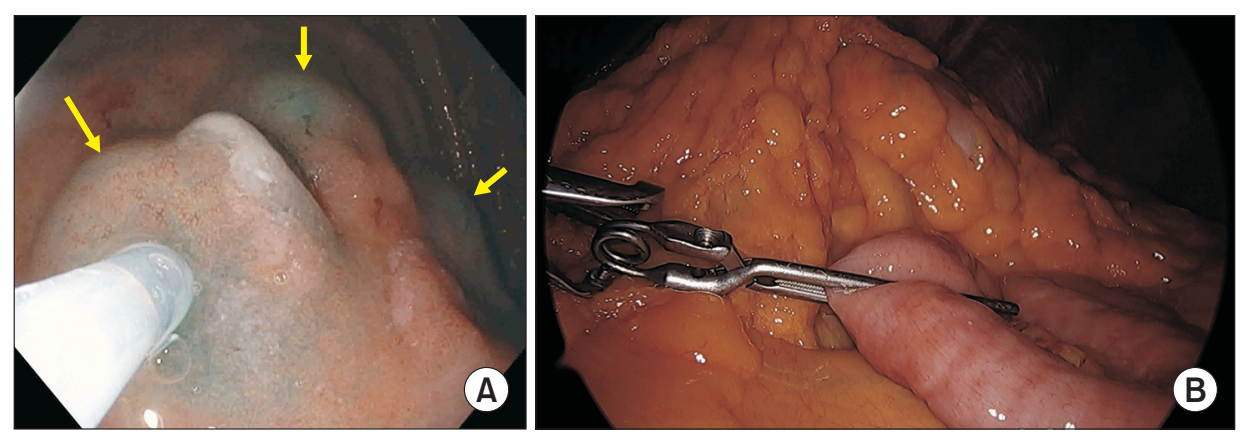

Fig. 2. Endoscopic injection of indocyanine green. (A) Submucosal injection of indocyanine green using endoscopic injection needle around the tumor. (B) Blockage of proximal jejunum using atraumatic forceps or clamps can prevent accidental inflation of the small bowels, which may interrupt operation, during endoscopic injection.

Furthermore, if the injected ICG leaks from the injection site, it can stain the operation field. The identification of the lymphatic structures and lymph nodes can be disturbed because the NIR signal of ICG is very strong and ICG is very difficult to be removed from stained tissues.

In contrast, submucosal injection via an endoscopic needle has more advantages given accurate localization of the tumor and less technical difficulties of injection depending on the location. ${ }^{21,23-27,30,31}$ Endoscopic injection has a risk of penetration of the gastric wall and spillage of the ICG into the peritoneal space, so some a learning period may be required for beginners.

Lymph node navigation is used for sentinel node navigation surgery, but it can also be used to evaluate and/or ensure complete wide lymph node dissection. Some clinical trials to evaluate new laparoscopic gastrectomy technologies tried to ensure adequate and complete lymph node dissection and opt to collect photographs of the operative field after completion of the lymph node dissection. ${ }^{33}$ NIR imaging may provide further information regarding whether ICG-stained lymph nodes were completely removed from the field. In this context, ICG injection does not have to be limited to peritumoral area but can be widely performed at multiple areas, i.e., several spots along the lesser curvature side and several spots along the greater curvature side, to cover all the main perigastric lymphatic pathways.

Injection methods and results are summarized in Table 1.

\section{ICG INJECTION AMOUNT}

NIR imaging is very sensitive to small amounts of ICG, and the NIR signal is not linearly correlated to the amount of ICG. A special characteristic of ICG is the "quenching effect", which refers to the phenomenon whereby the signal decreases with increasing concentrations of ICG because closely crowded ICG molecules absorb the NIR signal from nearby ICG molecules. Consequently, the NIR signal of ICG is the strongest at a concentration of $0.001 \sim 0.01 \mathrm{mg} / \mathrm{ml}$ and decreases as the concentration either increases or decreases (Fig. 3). ) $^{34,35}$ The default concentration obtained from commercially available ICG is 


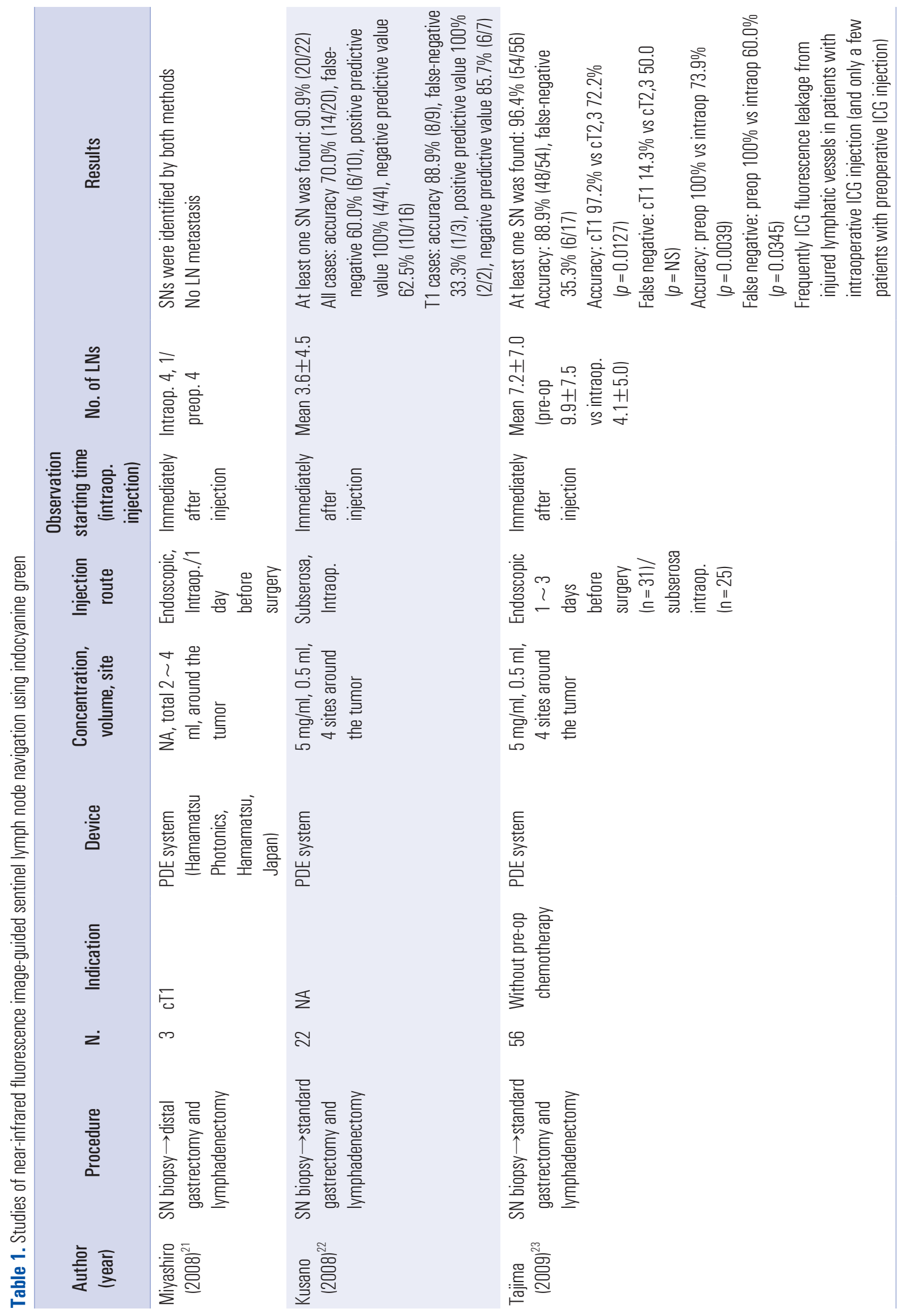




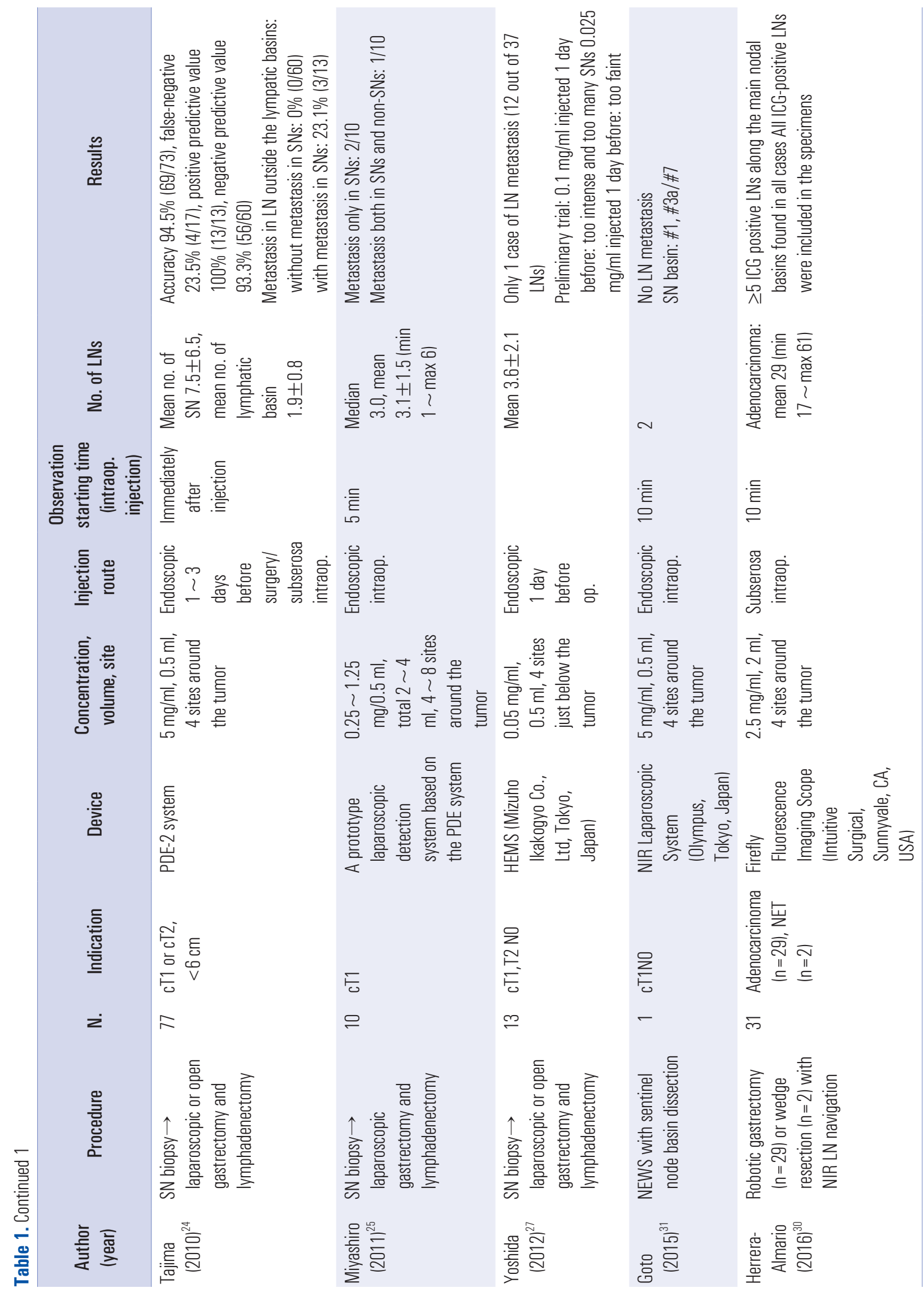




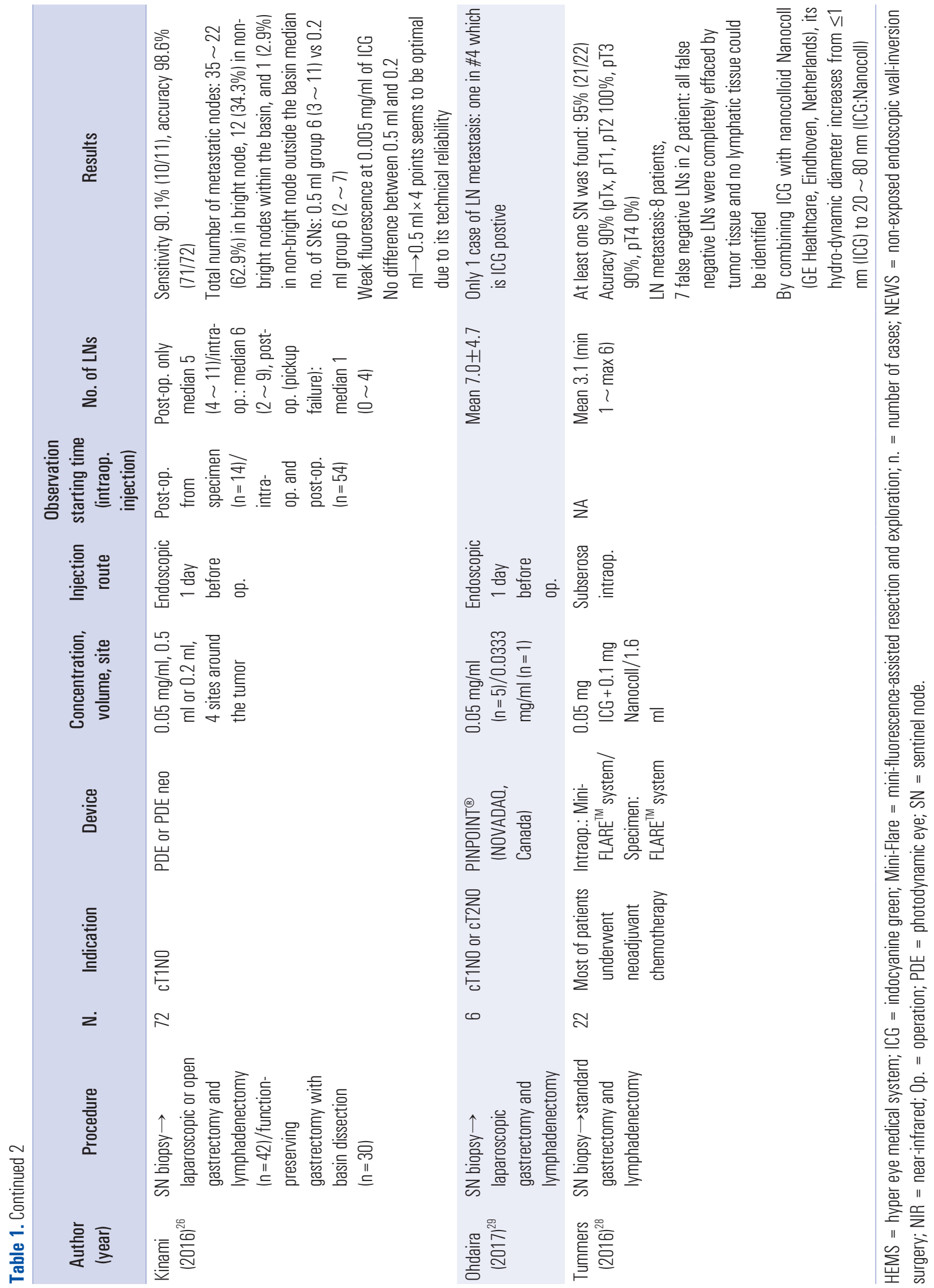



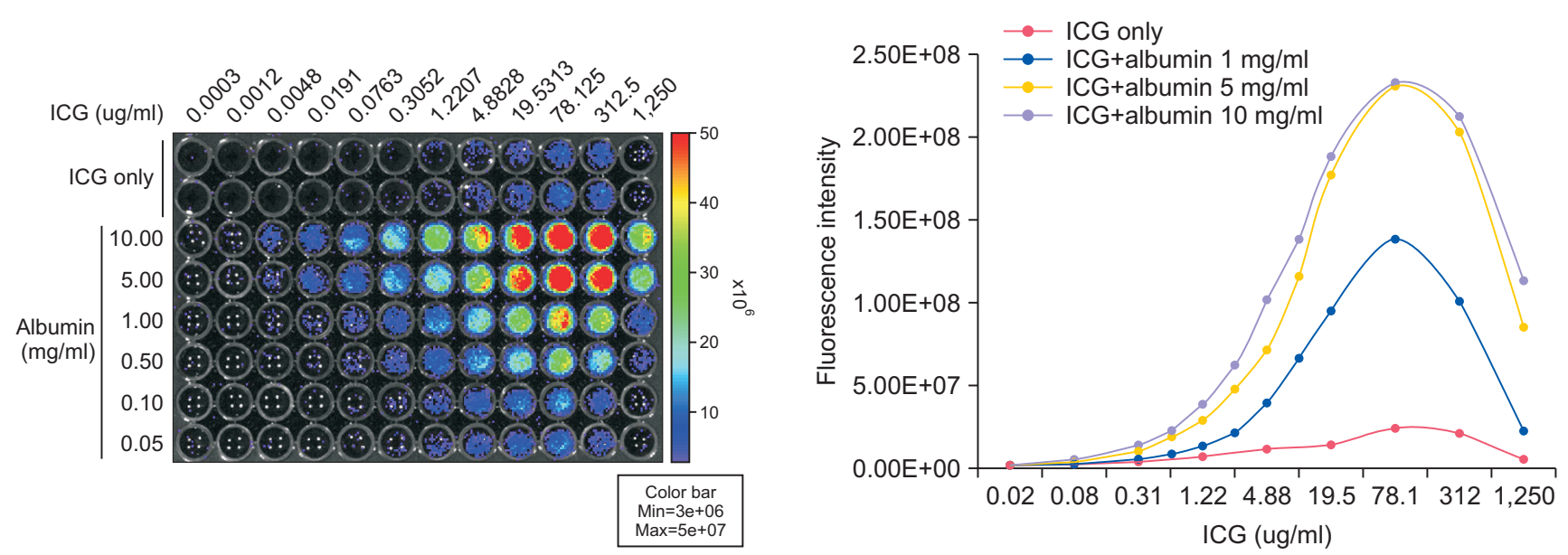

Fig. 3. Near-infrared fluorescence signal intensity of indocyanine green depending on the concentration of indocyanine green and albumin measure using the IVIS ${ }^{\circledR}$ spectrum in vivo imaging system (Xenogen Corporation, CA, USA).

either $5 \mathrm{mg} / \mathrm{ml}$ or $2.5 \mathrm{mg} / \mathrm{ml}$, and this concentration cannot provide any NIR signal unless it is diluted numerous times. Theoretically, if a high concentration of ICG is injected, a nearby lymph node cannot be detected before the concentration is diluted. Therefore, the amount or the concentration of injected ICG should be decided such that the final concentration after dilution of injected ICG in the stomach can achieve the concentration of the highest signal. Because ICG is a free molecule and is rapidly and widely diffused, an excessive concentration of ICG can highlight too many lymph nodes for use in sentinel node biopsy. In addition, ICG can diffuse to adjacent soft tissues surrounding lymph nodes and stain the operative field via spillage from soft tissues during dissection. The timing of injection may also affect the decision regarding the injection dosage, but there are not a sufficient number of studies to clarify this effect to date. Yoshida et al. injected ICG via the endoscopic method one day before the operation. In this study, the ICG fluorescence of a patient injected $100 \mathrm{mg} /$ $\mathrm{ml}$ was too intense and that of a patient injected $25 \mathrm{mg} / \mathrm{mL}$ was too faint. ${ }^{27}$

In the human body, a significant proportion of ICG is attached to albumin. Albumin stabilizes and maintains the distance between the ICG molecules, and different albumin concentrations can affect the NIR signal intensity of ICG. Once ICG powder is mixed with the provided solution, it starts to lose the NIR signal via bleaching. The signal can be lost after 24 hours in vitro; however, the signal persists for a couple of days after injection into living organs by attaching to albumin or via phagocytosis by macrophages. ${ }^{36}$

Regarding the volume of ICG solution, Kinami et al. injected $50 \mu \mathrm{g} / \mathrm{ml}$ of ICG 4 quadrants of the primary tumor on the day prior to the surgery and compared $0.5 \mathrm{ml}$ and $0.2 \mathrm{ml}$ of injection at each site in terms of the number of bright nodes. ${ }^{26}$
No differences in bright nodes were noted between the two groups [6 (range, 3 11) in the $0.5 \mathrm{ml}$ group and 6 (range, 2 7) in the $0.2 \mathrm{ml}$ group]. They determined that the adequate injection volume of ICG solution was $0.5 \mathrm{ml}$, and many other investigators used $0.5 \mathrm{ml}$ given its technical reliability.

\section{TIMING OF INJECTION}

The injection is performed either at the time of initiation of the operation or 1 day before operation (Table 1). The advantage of the intraoperative injection it that the injection can be made under visual control in the operative view to minimize spillage and control spillage when it occurs regardless of whether a subserosal or submucosal injection is performed. The length of time is required for diffusion of ICG from the injection site to the lymph nodes has been not well established, but the lymphatic vessels are often visualized immediately after injection. ${ }^{22,23}$ Here, 10 20 minutes may be a sufficient waiting time. ${ }^{25,26,31}$

Preoperative injection can be selected when endoscopy is not easily available in the operation theatre. Visualization of lymph nodes as well as lymphatic channels is regarded as an additional benefit compared with methods using radioisotopes. Although the lymphatic channels are more clearly visualized during the early period after intraoperative injection, it was also reported that lymphatic channels were visualized with an injection performed 1 day before operation. ${ }^{23}$ Some authors reported that the intraoperative injection resulted in frequent fluorescence leakage from injured lymphatic vessels and too many false positive lymph nodes in case of intraoperative injection. These researchers preferred pre-operative injection. ${ }^{23,27}$

Lymph nodes with NIR signals remaining one day after injection could be either sentinel lymph nodes or more distant 


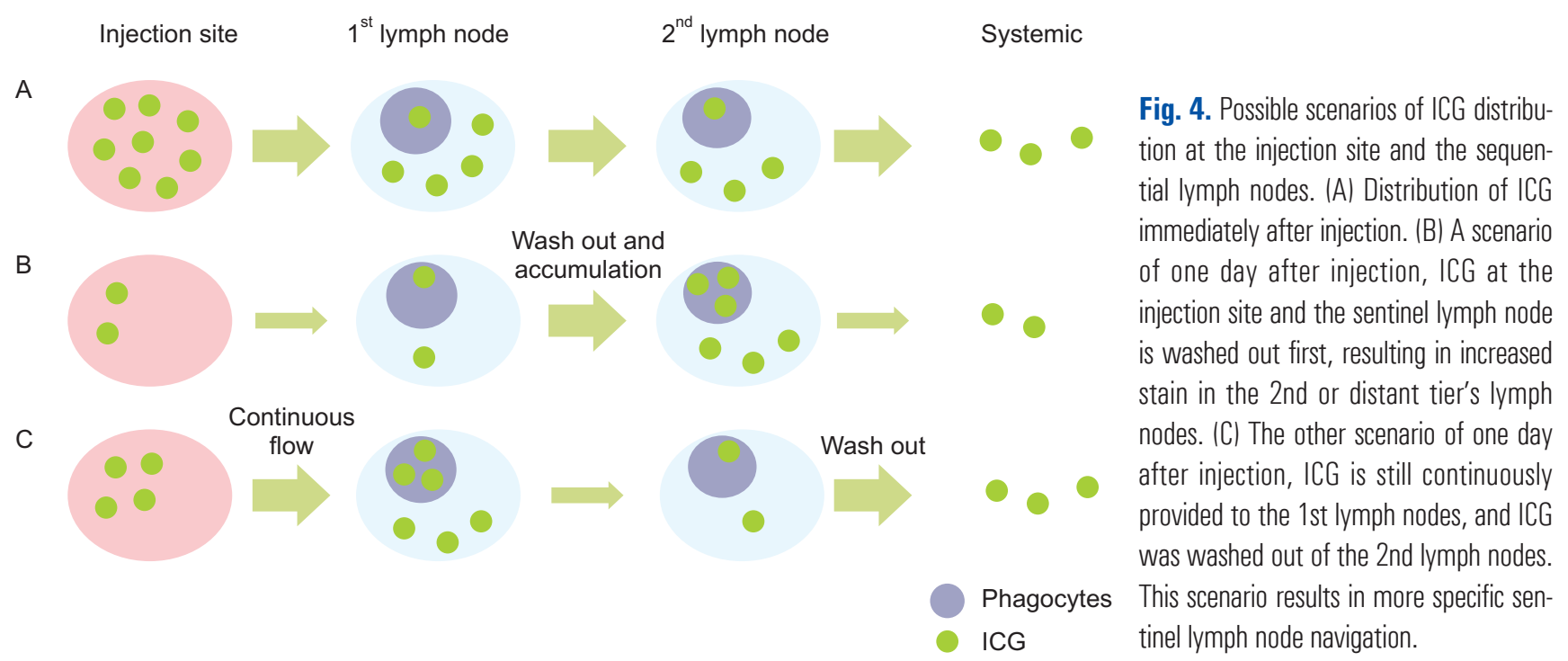

lymph nodes from the injection site. If the ICG is washed out sequentially from the injection site to the 1st tier lymph node and more distant lymph nodes, it is more likely that the bright lymph nodes are 2nd or 3rd tier lymph nodes after sentinel lymph nodes (Fig. 4B). On the other hand, if ICG at the injection site continuously provides ICG to the 1st lymph nodes and the ICG at the distal lymph nodes is drained, the remaining bright lymph nodes are more likely sentinel nodes (Fig. 4C). To date, the second scenario seems to better explain the results of previous reports indicating that the bright lymph nodes were located near the ICG injection site.

\section{NUMBER OF SENTINEL LYMPH NODES}

There is no solid consensus about the ideal number of sentinel lymph nodes in gastric cancer surgery. Some examples of number of lymph nodes include 2 2.8 when using the dye method $^{37,38}$ and 3.3 4.1 when using dual tracers. ${ }^{14,39}$ To increase the sensitivity of the identification of sentinel lymph nodes and reduce false negative nodes, which can be the most important value in terms of oncologic safety, an increased number of sentinel lymph nodes can be acceptable. However, there are practical limitations regarding the resection and evaluation of too many lymph nodes determine whether cancer cells are present inside those lymph nodes. Furthermore, basin dissection rather than node selection is recommended for gastric cancer considering the false negative lymph nodes in the same basin of false positive lymph nodes. If ICG spreads to too many basins, reducing the number of sentinel lymph nodes or tailoring the extent of the dissection can be meaningless. Ryu et al. performed a meta-analysis of the 46 studies which used dye, radioisotope or both tracer, and reported that a sen- sitivity of the pooled estimate of studies became significantly improved when the number of the sentinel node is 5 or more compares to 4 or less number of the sentinel nodes $(92.6 \%$ vs $82.3 \%)$ and there was no further improvement of sensitivity as the number of the sentinel node increases. ${ }^{40}$ Based on this result, a Korean multicenter clinical trial (SENORITA) suggested an optimal number of sentinel lymph node as at least $5 .^{41}$

Tajima et al. endoscopically injected $0.5 \%(5 \mathrm{mg} / \mathrm{ml})$ of ICG at 4 sites around the tumor using a $0.5-\mathrm{ml}$ injection volume at each site 1 3 days before operation, and 7.2 \pm 7.0 (1 30) lymph nodes were brightened. ${ }^{23}$ Miyashiro et al. injected $0.5 \sim 2.5 \mathrm{mg} /$ $\mathrm{ml}$ of ICG at 4 sites around the tumor using a $0.5-\mathrm{ml}$ of injection volume by intraoperative endoscopy, and $3.1 \pm 1.5$ lymph nodes were identified. Yoshida et al. injected $0.05 \mathrm{mg} / \mathrm{ml}$ of ICG using a $0.5-\mathrm{ml}$ volume via endoscopy one day before surgery, and 3.6 \pm 2.1 lymph nodes were identified. Kinami et al. injected ICG in a similar manner, and the median number of lymph nodes was 6 (3 11). These limited results suggest that the high concentration can affect many lymph nodes, and the $0.05 \mathrm{mg} / \mathrm{ml}$ concentration and $0.5-\mathrm{ml}$ volume can serve as a reference dosage for further studies.

However, the optimal dosage depends on different factors, including timing of injection, amount of fat in the perigastric lymphatic basins, and different imaging devices with different sensitivities. These factors should be further studied.

\section{FUTURE OF NODE NAVIGATION USING NIR IMAGING}

A fundamental question about sentinel lymph node navigation is whether it can solve current concerns about false negative issues and can be established as a standard treatment of 
gastric cancer. ${ }^{42-45}$ Further development of the basin dissection concept and strategies to chase the lymphatic pathway and lymph nodes with NIR signals may provide additional solutions.

In addition, the advantages of NIR imaging over current optimal options, such as a dual method with vital dye and radioisotope, include improved visualization and sensitivity compared with vital dyes and lack of radioactive concerns, supporting the possibility of replacing the dual method with single NIR imaging. Otherwise, NIR imaging could be justified if it can provide optimal safety for sentinel node navigation surgery when combined with radioisotopes. Skubleny et al. recently published a meta-analysis of sentinel lymph node biopsy using indocyanine green and infrared or fluorescent imaging of gastric cancer. ${ }^{46}$ The sensitivity of this meta-analysis was relatively low as $87 \%(0.80 \sim 0.93)$ in pooled analysis. The study also reported sensitivity of the studies using infrared electronic endoscopic as $97 \%$ and that of the studies using NIR fluorescent imaging systems as $72 \% .^{24,28}$ However, only 2 studies with NIR imaging system were included in this study, and the studies included high proportion of cases with advanced gastric cancer. A large comparison study to compare ICG NIR imaging and the dual method is needed, too. Some small studies of breast cancer and gynecologic cancers suggested non-inferiority of NIR imaging compared with radioisotopes. ${ }^{47-49}$ Given the low incidence of lymph node metastasis from early gastric cancer, thousands of cases may be required to obtain sufficient statistical power to obtain a high level of evidence.

A tool for guidance or evaluation of wide radical lymph node dissection is another possible application of ICG NIR imaging technology more for advanced gastric cancer cases. In particular, the technology may help surgeons in Western countries who are not familiar with wide lymph node dissection. At the present time, this technique can be regarded as an additional tool for these purposes because false positive and false negative results are inferior in cases of advanced cancer compared with early gastric cancer cancers. ${ }^{22,23}$ This finding is probably due to blocking of lymphatic channels and lymph nodes by tumor cells. On the other hand, some researchers may be concerned about the possibility of unnecessary extensive surgery and subsequent complications, which could be caused by too sensitive and wide false positive ICG signals. Therefore, whether the area with ICG NIR signal can represent all the lymphatic structures within the area of standard lymph node dissection, whether further dissection of area with ICG signal outside the boundary of the standard lymph node dissection, and whether this technology can contribute the patients survival should be further studied in clinical trials.

Research is ongoing to overcome the limitations of ICG, such as the rapid and wide distribution and non-specificity for the cancer. Some of examples include the use of modified cyanine dyes to increase the size to limit the diffusion of the tracer and monoclonal antibodies or affibodies tagged with NIR dye, which are specific to the molecular targets. ${ }^{16,28,36,50-53}$ Multi-functional dyes, which can be detected by multiple diagnostic tools, including pre-operative CT or MRI, NIR imaging systems, and gamma-probe detectors for radioisotopes, may be helpful to plan and implement comprehensive planning and surgeries for gastric cancer. ${ }^{54,55}$

\section{CONCLUSIONS}

NIR imaging investigations of lymph node navigation in gastric cancer surgery is a promising tool with possible advantages compared with vital dyes or radioisotopes. Given the characteristics of ICG, including quenching and fast diffusion, diluted concentrations are suggested for sentinel lymph node biopsy. A large-scale study is required to evaluate the accuracy of prediction of lymph node metastasis, and this technique should be compared with dual methods using vital dyes and radioisotopes.

\section{REFERENCES}

1) Marescaux J, Diana M. Next step in minimally invasive surgery: hybrid image-guided surgery. J Pediatr Surg 2015;50:30-36.

2) Bernhardt S, Nicolau SA, Soler L, Doignon C. The status of augmented reality in laparoscopic surgery as of 2016. Med Image Anal 2017;37:66-90.

3) Paulus CJ, Haouchine N, Kong SH, Soares RV, Cazier D, Cotin S. Handling topological changes during elastic registration : Application to augmented reality in laparoscopic surgery. Int J Comput Assist Radiol Surg 2017;12:461-470.

4) Herbort CP, LeHoang P, Guex-Crosier Y. Schematic interpretation of indocyanine green angiography in posterior uveitis using a standard angiographic protocol. Ophthalmology 1998;105:432440.

5) Raabe A, Beck J, Gerlach R, Zimmermann M, Seifert V. Nearinfrared indocyanine green video angiography: a new method for intraoperative assessment of vascular flow. Neurosurgery 2003;52: 132-139; discussion 139.

6) Reuthebuch O, Haussler A, Genoni M, et al. Novadaq SPY: intraoperative quality assessment in off-pump coronary artery bypass grafting. Chest 2004;125:418-424.

7) Benson RC, Kues HA. Fluorescence properties of indocyanine green as related to angiography. Phys Med Biol 1978;23:159-163.

8) Desmettre T, Devoisselle JM, Mordon S. Fluorescence properties and metabolic features of indocyanine green (ICG) as related to angiography. Surv Ophthalmol 2000;45:15-27. 
9) Ris F, Hompes R, Cunningham C, et al. Near-infrared (NIR) perfusion angiography in minimally invasive colorectal surgery. Surg Endosc 2014;28:2221-2226.

10) Boni L, David G, Dionigi G, Rausei S, Cassinotti E, Fingerhut A. Indocyanine green-enhanced fluorescence to assess bowel perfusion during laparoscopic colorectal resection. Surg Endosc 2016; 30:2736-2742.

11) Liu YY, Liao CH, Diana M, et al. Near-infrared cholecystocholangiography with direct intragallbladder indocyanine green injection: preliminary clinical results. Surg Endosc 2018;32:1506-1514.

12) Boogerd LSF, Handgraaf HJM, Huurman VAL, et al. The Best Approach for Laparoscopic Fluorescence Cholangiography: Overview of the Literature and Optimization of Dose and Dosing Time. Surg Innov 2017;24:386-396.

13) Ishizawa T, Saiura A, Kokudo N. Clinical application of indocyanine green-fluorescence imaging during hepatectomy. Hepatobiliary Surg Nutr 2016;5:322-328.

14) Park DJ, Kim HH, Park YS, et al. Simultaneous indocyanine green and (99m)Tc-antimony sulfur colloid-guided laparoscopic sentinel basin dissection for gastric cancer. Ann Surg Oncol 2011;18:160165.

15) Vahrmeijer AL, Hutteman M, van der Vorst JR, van de Velde CJ, Frangioni JV. Image-guided cancer surgery using near-infrared fluorescence. Nat Rev Clin Oncol 2013;10:507-518.

16) Kong SH, Noh YW, Suh YS, et al. Evaluation of the novel nearinfrared fluorescence tracers pullulan polymer nanogel and indocyanine green/gamma-glutamic acid complex for sentinel lymph node navigation surgery in large animal models. Gastric Cancer 2015;18:55-64.

17) Hachey KJ, Gilmore DM, Armstrong KW, et al. Safety and feasibility of near-infrared image-guided lymphatic mapping of regional lymph nodes in esophageal cancer. J Thorac Cardiovasc Surg 2016;152:546-554.

18) Currie AC, Brigic A, Thomas-Gibson S, et al. A pilot study to assess near infrared laparoscopy with indocyanine green (ICG) for intraoperative sentinel lymph node mapping in early colon cancer. Eur J Surg Oncol 2017;43:2044-2051.

19) Rocha A, Dominguez AM, Lecuru F, Bourdel N. Indocyanine green and infrared fluorescence in detection of sentinel lymph nodes in endometrial and cervical cancer staging - a systematic review. Eur J Obstet Gynecol Reprod Biol 2016;206:213-219.

20) Yuan B, Chen N, Zhu Q. Emission and absorption properties of indocyanine green in Intralipid solution. J Biomed Opt 2004;9: 497-503.

21) Miyashiro I, Miyoshi N, Hiratsuka M, et al. Detection of sentinel node in gastric cancer surgery by indocyanine green fluorescence imaging: comparison with infrared imaging. Ann Surg Oncol 2008;15:1640-1643.

22) Kusano M, Tajima Y, Yamazaki K, Kato M, Watanabe M, Miwa M. Sentinel node mapping guided by indocyanine green fluorescence imaging: a new method for sentinel node navigation surgery in gastrointestinal cancer. Dig Surg 2008;25:103-108.

23) Tajima Y, Yamazaki K, Masuda Y, et al. Sentinel node mapping guided by indocyanine green fluorescence imaging in gastric cancer. Ann Surg 2009;249:58-62.

24) Tajima Y, Murakami M, Yamazaki K, et al. Sentinel node mapping guided by indocyanine green fluorescence imaging during laparoscopic surgery in gastric cancer. Ann Surg Oncol 2010;17:17871793.

25) Miyashiro I, Kishi K, Yano M, et al. Laparoscopic detection of sentinel node in gastric cancer surgery by indocyanine green fluorescence imaging. Surg Endosc 2011;25:1672-1676.

26) Kinami S, Oonishi T, Fujita J, et al. Optimal settings and accuracy of indocyanine green fluorescence imaging for sentinel node biopsy in early gastric cancer. Oncol Lett 2016;11:4055-4062.

27) Yoshida M, Kubota K, Kuroda J, et al. Indocyanine green injection for detecting sentinel nodes using color fluorescence camera in the laparoscopy-assisted gastrectomy. J Gastroenterol Hepatol 2012;27 Suppl 3:29-33.

28) Tummers QR, Boogerd LS, de Steur WO, et al. Near-infrared fluorescence sentinel lymph node detection in gastric cancer: A pilot study. World J Gastroenterol 2016;22:3644-3651.

29) Herrera-Almario G, Patane M, Sarkaria I, Strong VE. Initial report of near-infrared fluorescence imaging as an intraoperative adjunct for lymph node harvesting during robot-assisted laparoscopic gastrectomy. J Surg Oncol 2016;113:768-770.

30) Ohdaira H, Yoshida M, Okada S, Tsutsui N, Kitajima M, Suzuki Y. New method of indocyanine green fluorescence sentinel node mapping for early gastric cancer. Ann Med Surg (Lond) 2017;20: 61-65.

31) Goto O, Takeuchi H, Kawakubo H, et al. First case of non-exposed endoscopic wall-inversion surgery with sentinel node basin dissection for early gastric cancer. Gastric Cancer 2015;18:434439.

32) Lee JH, Ryu KW, Kim CG, et al. Comparative study of the subserosal versus submucosal dye injection method for sentinel node biopsy in gastric cancer. Eur J Surg Oncol 2005;31:965-968.

33) Hur H, Lee HY, Lee HJ, et al. Efficacy of laparoscopic subtotal gastrectomy with D2 lymphadenectomy for locally advanced gastric cancer: the protocol of the KLASS-02 multicenter randomized controlled clinical trial. BMC Cancer 2015;15:355.

34) Yang $\mathrm{H}-\mathrm{K}$, Kong $\mathrm{S}-\mathrm{H}$, SuH Y-S, Lee H-J. ICG-Enhanced NIR Fluorescence Imaging in Laparoscopic Gastric Cancer Surgery [Internet]. Schramberg, Germany: EndoPress; c2018 [cited on 2018 May 6]. Available from: https://www.karlstorz.com/cps/rde/ xbcr/karlstorz_assets/ASSETS/3544618.pdf.

35) Takeyama H, Hata T, Nishimura J, et al. A novel endoscopic fluorescent clip visible with near-infrared imaging during laparoscopic surgery in a porcine model. Surg Endosc 2014;28:1984-1990.

36) Noh YW, Kong SH, Choi DY, et al. Near-infrared emitting poly- 
mer nanogels for efficient sentinel lymph node mapping. ACS Nano 2012;6:7820-7831.

37) Cozzaglio L, Bottura R, Di Rocco M, Gennari L, Doci R. Sentinel lymph node biopsy in gastric cancer: possible applications and limits. Eur J Surg Oncol 2011;37:55-59.

38) Rabin I, Chikman B, Lavy R, et al. The accuracy of sentinel node mapping according to $\mathrm{T}$ stage in patients with gastric cancer. Gastric Cancer 2010;13:30-35.

39) Kitagawa Y, Saikawa Y, Takeuchi H, et al. Sentinel node navigation in early stage gastric cancer--updated data and current status. Scand J Surg 2006;95:256-259.

40) Ryu KW, Eom BW, Nam BH, et al. Is the sentinel node biopsy clinically applicable for limited lymphadenectomy and modified gastric resection in gastric cancer? A meta-analysis of feasibility studies. J Surg Oncol 2011;104:578-584.

41) Lee YJ, Jeong SH, Hur H, et al. Prospective Multicenter Feasibility Study of Laparoscopic Sentinel Basin Dissection for Organ Preserving Surgery in Gastric Cancer: Quality Control Study for Surgical Standardization Prior to Phase III Trial. Medicine (Baltimore) 2015;94:e1894.

42) Eom BW, Kim YI, Yoon HM, et al. Current status and challenges in sentinel node navigation surgery for early gastric cancer. Chin J Cancer Res 2017;29:93-99.

43) Miyashiro I. What is the problem in clinical application of sentinel node concept to gastric cancer surgery? J Gastric Cancer 2012;12: 7-12.

44) Miyashiro I, Hiratsuka M, Sasako M, et al. High false-negative proportion of intraoperative histological examination as a serious problem for clinical application of sentinel node biopsy for early gastric cancer: final results of the Japan Clinical Oncology Group multicenter trial JCOG0302. Gastric Cancer 2014;17:316-323.

45) Kitagawa Y, Takeuchi H, Takagi Y, et al. Sentinel node mapping for gastric cancer: a prospective multicenter trial in Japan. J Clin Oncol 2013;31:3704-3710.

46) Skubleny D, Dang JT, Skulsky S, et al. Diagnostic evaluation of sentinel lymph node biopsy using indocyanine green and infrared or fluorescent imaging in gastric cancer: a systematic review and meta-analysis. Surg Endosc 2018;32:2620-2631.

47) Ballardini B, Santoro L, Sangalli C, et al. The indocyanine green method is equivalent to the (9)(9)mTc-labeled radiotracer method for identifying the sentinel node in breast cancer: a concordance and validation study. Eur J Surg Oncol 2013;39:1332-1336.

48) Di Martino G, Crivellaro C, De Ponti E, et al. Indocyanine Green versus Radiotracer with or without Blue Dye for Sentinel Lymph Node Mapping in Stage >IB1 Cervical Cancer (>2 cm). J Minim Invasive Gynecol 2017;24:954-959.

49) Soergel P, Hertel H, Nacke AK, Klapdor R, Derlin T, Hillemanns P. Sentinel Lymphadenectomy in Vulvar Cancer Using NearInfrared Fluorescence From Indocyanine Green Compared With Technetium 99m Nanocolloid. Int J Gynecol Cancer 2017;27:805812.

50) Ohnishi S, Lomnes SJ, Laurence RG, Gogbashian A, Mariani G, Frangioni JV. Organic alternatives to quantum dots for intraoperative near-infrared fluorescent sentinel lymph node mapping. Mol Imaging 2005;4:172-181.

51) Lee SB, Hassan M, Fisher R, et al. Affibody molecules for in vivo characterization of HER2-positive tumors by near-infrared imaging. Clin Cancer Res 2008;14:3840-3849.

52) Zhang RR, Schroeder AB, Grudzinski JJ, et al. Beyond the margins: real-time detection of cancer using targeted fluorophores. Nat Rev Clin Oncol 2017;14:347-364.

53) Haque A, Faizi MSH, Rather JA, Khan MS. Next generation NIR fluorophores for tumor imaging and fluorescence-guided surgery: A review. Bioorg Med Chem 2017;25:2017-2034.

54) Kim H, Lee SK, Kim YM, et al. Fluorescent iodized emulsion for pre- and intraoperative sentinel lymph node imaging: validation in a preclinical model. Radiology 2015;275:196-204.

55) Kim YH, Lee YJ, Park JH, et al. Early gastric cancer: feasibility of CT lymphography with ethiodized oil for sentinel node mapping. Radiology 2013;267:414-421. 